04

\title{
Ускорение потока пылевой плазмы в азимутальном электрическом и радиальном магнитном полях
}

\author{
(C) A.P. Каримов ${ }^{1,2}$, C.A. Терехов ${ }^{2, \uparrow, ~ А . Е . ~ Ш и к а н о в ~}{ }^{2}$ \\ ${ }^{1}$ Объединенный институт высоких температур РАН, Москва, Россия \\ ${ }^{2}$ Национальный исследовательский ядерный университет „МИФИ“, Москва, Россия \\ ฯ E-mail: SATerekhov@mephi.ru
}

Поступило в Редакцию 18 апреля 2019г.

В окончательной редакции 25 декабря 2019 г.

Принято к публикации 27 декабря 2019г.

\begin{abstract}
В рамках модели холодной гидродинамики изучается ускорение потока пылевой плазмы в азимутальном электрическом и радиальном магнитном полях. Приведенные результаты показывают, что существуют условия, при которых макроскопические частицы захватываются и ускоряются плазменным потоком.
\end{abstract}

Ключевые слова: магнитные поля, пылевая плазма, нелинейные волны, ускоритель.

DOI: 10.21883/PJTF.2020.07.49215.17843

Высокоскоростные плазменные потоки, состоящие из электронов, ионов и заряженных макроскопических частиц, представляют интерес для различных технических приложений [1-4]. Для ускорения таких потоков используют рельсовые ускорители, основанные на непосредственном преобразовании электромагнитной энергии импульса тока в кинетическую энергию ускоряемых тел за счет действия силы Ампера на плазменный сгусток, возникающий в канале ускорителя [5]. При этом величина достижимой скорости метания макротел определяется только процессами накопления и выделения электрической энергии [5-8]. Другой причиной, ограничивающей максимальную скорость, является развитие неустойчивости плазменного поршня, проявляющейся при изменении тока в цепи ускорителя [7]. Поэтому представляет интерес рассмотрение альтернативных способов ускорения плазменных потоков, содержащих макроскопические заряженные частицы.

В настоящей работе рассматривается ускорение заряженных макроскопических частиц, входящих в состав пылевой плазмы, в результате их захвата вращающимся плазменным потоком, который, попадая в область скрещенных магнитных полей, изображенную на рис. 1, $a$, ускоряется за счет передачи импульса между макроскопическими степенями свободы потока плазмы [9-11]. При этом следует отметить, что исследуется вариант нескоррелированных пылевых частиц, когда коллективное воздействие заряженных пылинок на процессы, происходящие в плазме, ничтожно, т.е. плотность заряда пыли мала по сравнению с плотностью плазмы [12]:

$$
Z_{d} n_{d} \ll n_{e} .
$$

Поскольку заряд пылевой частицы зависит от ее размеров, данное условие также ограничивает ее размеры. В противном случае возникают эффекты дальнего порядка, когда уже невозможно ограничиться захватом отдельных пылевых частиц в основной плазменный поток [13].

В рассматриваемом случае магнитное поле имеет постоянную радиальную компоненту $\mathbf{B}_{r_{0}}$ и меняющуюся во времени аксиальную составляющую $\mathbf{B}_{z_{0}}(t)$, которая порождает азимутальное электрическое поле, закручивающее поток ионов $\mathbf{j}_{i \varphi}$ в одну сторону, а потоки электронов $\mathbf{j}_{e \varphi}$ и отрицательно заряженных макрочастиц $\mathbf{j}_{d \varphi}$ в противоположную сторону $\left(\mathbf{F}_{i}\right.$ и $\mathbf{F}_{e, d}-$ силы, которые действуют на потоки ионов, электронов и макрочастиц, показывающие, что эти потоки ускоряются в одну сторону). Взаимодействие данных токов с постоянным радиальным магнитным полем $\mathbf{B}_{r_{0}}$ приводит к ускорению всего плазменного потока в аксиальном направлении. Техническая реализация этой идеи представлена на рис. $1, b[10]$. Кроме того, такая конфигурация магнитного поля должна усилить обмен импульсом/энергией между макроскопическими степенями свободы потока из-за генерации собственного электрического поля внутри потока [14].

Чтобы описать процесс ускорения в схеме, изображенной на рис. 1, $a$, мы рассмотрим аксиально-симметричное течение холодного потока пылевой плазмы, начальный поперечный размер которой $R_{0}$ удовлетворяет условию $a \ll R_{0} \ll b$, где $a$ и $b-$ внутренний и внешний радиусы магнитной системы. Представим электрическое и магнитное поля в виде сумм внешней и собственной составляющих:

$$
\begin{aligned}
& \mathbf{E}=\mathbf{E}_{0}+\mathbf{E}^{*}, \\
& \mathbf{B}=\mathbf{B}_{0}+\mathbf{B}^{*},
\end{aligned}
$$

где собственные электрические $\mathbf{E}^{*}$ и магнитные $\mathbf{B}^{*}$ поля определяются динамическими процессами в самой 

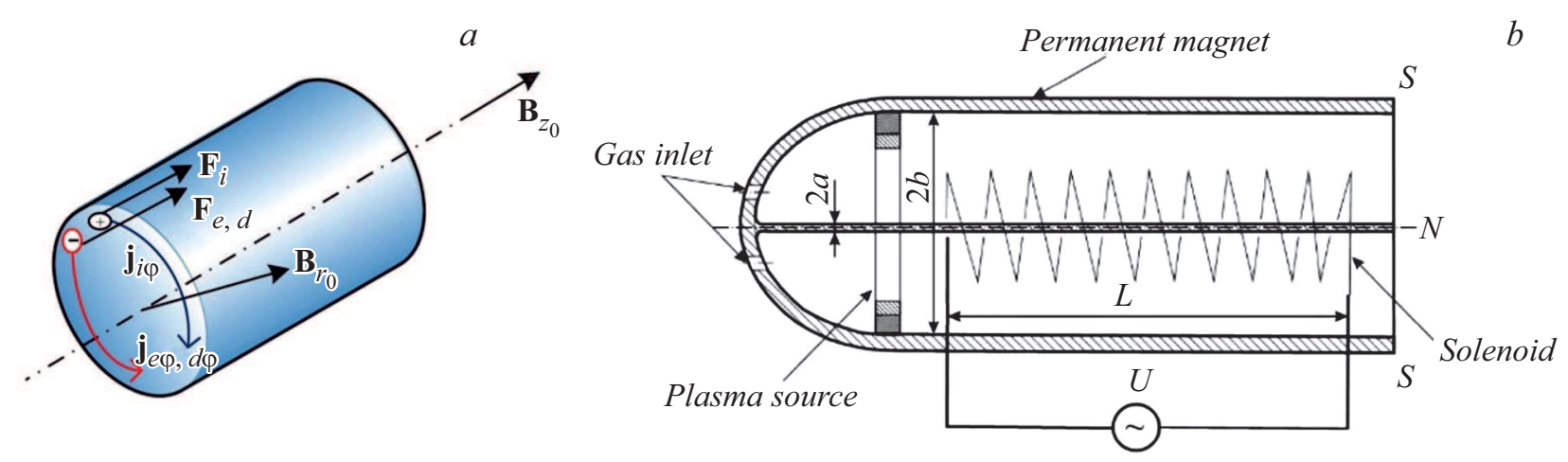

Рис. 1. $a-$ схема участка ускорения пылевой плазмы; $b-$ конструкция ускорителя.

плазменной среде, а внешнее магнитное поле есть

$$
\mathbf{B}_{0}=B_{r_{0}} \mathbf{e}_{r}+B_{z_{0}} \mathbf{e}_{z}
$$

Здесь $B_{r_{0}}=$ const - постоянное радиальное магнитное поле известной величины, а известная функция $B_{z 0}(t)$ определяет изменение по времени аксиальной составляющей магнитного поля. Отметим, что в отличие от традиционных плазменных ускорителей, где внешние электрическое $\mathbf{E}_{0}$ и магнитное $\mathbf{B}_{0}$ поля независимы и могут создаваться разными техническими способами, в нашем случае $\mathbf{E}_{0}$ порождается $\mathbf{B}_{0}$. Действительно, из выражения индукции магнитного поля, записанного для внешнего магнитного поля $\mathbf{B}_{0}$ в интегральной форме

$$
\int_{\gamma} \mathbf{E}_{0} d \mathbf{l}=\int_{S \gamma} \mathbf{B}_{0} d \mathbf{S},
$$

следует, что внешнее электрическое поле имеет только азимутальную составляющую

$$
E_{\varphi_{0}}=-\frac{r}{2} \partial_{t} B_{z_{0}}
$$

Пренебрегая тепловым движением компонент, будем описывать собственные параметры плазмы, включая электрическое $\mathbf{E}^{*}$ и магнитное $\mathbf{B}^{*}$ поля, уравнениями холодной гидродинамики. Рассмотрим случай, в котором собственный ток проводимости скомпенсирован током смещения, что реализуется при $\nabla \times \mathbf{B}^{*}=0$ (обсуждение применимости этого приближения дано в $[9,10])$. Тогда, пренебрегая пространственной зависимостью плотностей $n_{s}$, распределение скорости $\mathbf{v}_{s}$ и электрического поля можно представить в виде

$$
\begin{gathered}
n_{s}=n_{s}(t), \quad \mathbf{v}_{s}=r A_{s}(t) \mathbf{e}_{r}+r C_{s}(t) \mathbf{e}_{\varphi}+r D_{s}(t) \mathbf{e}_{z}, \\
\mathbf{E}^{*}=r \varepsilon_{r}(t) \mathbf{e}_{r}+r \varepsilon_{\varphi}(t) \mathbf{e}_{\varphi}+r \varepsilon_{z}(t) \mathbf{e}_{z},
\end{gathered}
$$

где $s=e, i, d$, а функции $A_{s}(t), C_{s}(t)$ и $D_{s}(t)$ соответствуют радиальной, азимутальной и аксиальной компонентам скорости $s$-й компоненты потока. Интересно отметить, что, согласно обобщенной теореме Джинса, данные зависимости являются следствием представления функции распределения в виде

$$
\begin{gathered}
f_{s}=f_{s}\left(I_{x s}, I_{y s}\right), \\
I_{l s}=a_{l s}(t) v_{x}+b_{l x}(t) v_{y}+c_{l s}(t) x+d_{l s}(t) y+h_{l s}(t),
\end{gathered}
$$

где $l=x, y$, а функции $a_{l s}(t), b_{l s}(t), c_{l s}(t), d_{l s}(t)$ и $h_{l s}(t)$ определяются подстановкой в уравнения Власова-Максвелла $[15,16]$. Однако проще сразу подставить (6), (7) в стандартные уравнения холодной гидродинамики и получить следующую систему нелинейных дифференциальных уравнений, записанную в безразмерной форме [11]:

$$
\begin{gathered}
\frac{d n_{s}}{d t}+2 n_{s} A_{s}=0, \\
\frac{d A_{s}}{d t}+A_{s}^{2}-C_{s}^{2}=\mu_{s}\left[\varepsilon_{r}+C_{s}\left(B_{z 0}+B_{z}^{*}\right)\right], \\
\frac{d C_{s}}{d t}+2 A_{s} C_{s}=\mu_{s}\left[\varepsilon_{\varphi}-\frac{1}{2} \frac{d B_{z_{0}}}{d t}+D_{s} B_{r_{0}}-A_{s}\left(B_{z_{0}}+B_{z}^{*}\right)\right], \\
\frac{d D_{s}}{d t}+A_{s} D_{s}=\mu_{s}\left[\varepsilon_{z}-C_{s} B_{r_{0}}\right], \\
\frac{d \varepsilon_{r}}{d t}=n_{e}\left(A_{e}-A_{i}\right)+Z_{d} n_{d}\left(A_{d}-A_{i}\right)-2 \varepsilon_{r} A_{i}, \\
\frac{d \varepsilon_{\varphi}}{d t}=n_{e}\left(C_{e}-C_{i}\right)+Z_{d} n_{d}\left(C_{d}-C_{i}\right)-2 \varepsilon_{r} C_{i}, \\
\frac{d \varepsilon_{z}}{d t}=n_{e}\left(D_{e}-D_{i}\right)+Z_{d} n_{d}\left(D_{d}-D_{i}\right)-2 \varepsilon_{r} D_{i}, \\
\frac{d B_{z}^{*}}{d t}=-2 \varepsilon_{\varphi},
\end{gathered}
$$

где введены следующие безразмерные параметры: $\mu_{i}=m_{e} / m_{i}, \mu_{e}=-1$ и $\mu_{d}=-Z_{d} m_{e} / m_{d} \quad\left(\mu_{e}-\right.$ масса электрона, $\mu_{i}$ - масса иона, $\mu_{d}$ и $-Z_{d}-$ масса и зарядовое число макроскопической частицы соответственно). В данной задаче в качестве характерного масштаба плотностей, времени и пространственного размера использовалась начальная электронная плотность $n_{e_{0}}$, обратная плазменная частота $\omega_{p e}=\left(4 \pi n_{e_{0}} e^{2} / m_{e}\right)^{1 / 2}$, начальный радиус плазменного потока $R_{0}$ соответственно. При этом скорости были обезразмерены на величину $R_{0} \omega_{p e}$, 

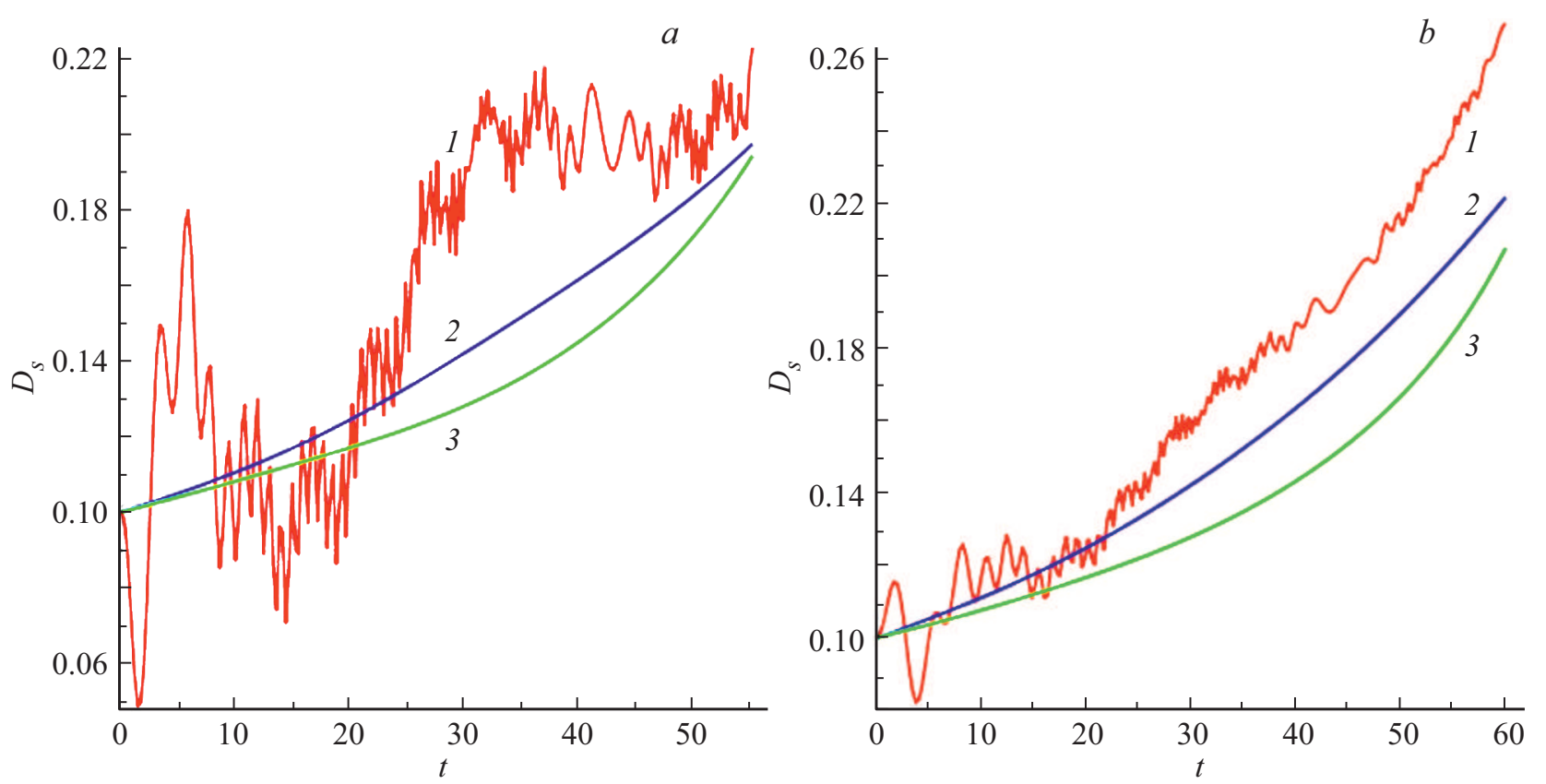

Рис. 2. Временны́е зависимости $D_{e} \quad(1), \quad D_{i} \quad(2) \quad$ и $\quad D_{d} \quad(3) \quad$ для $A_{e}(0)=A_{i}(0)=-10^{-2}, \quad A_{d}=-7 \cdot 10^{-3}$, $-C_{e}(0)=-C_{d}(0)=C_{i}(0)=10^{-3}, D_{e}(0)=D_{i}(0)=D_{d}(0)=10^{-1}$ в случае $B_{r_{0}}=-1$ и $B_{z_{0}}=-1(a)$ и в случае $B_{r_{0}}=-1$ и $B_{z_{0}}=-0.25 t(b)$.

электрическое поле - на $4 \pi e n_{e_{0}} R_{0}$, а магнитное поле на $4 \pi e n_{e_{0}} R_{0} c /\left(R_{0} \omega_{p e}\right)$.

Чтобы показать принципиальную возможность захвата и ускорения макроскопических частиц из пылевой плазмы в рамках модели (8), рассмотрим поток аргоновой плазмы с параметрами макрочастиц, описанными в работах [13,17-19]. В такой плазме присутствуют заряженные макроскопические аргоновые кластеры, имеющие массу $m_{d}=10^{3} m_{p}\left(m_{p}\right.$ - масса протона), характерный размер кластера $a=6 \cdot 10^{-7} \mathrm{~m}$ и величину отрицательного зарядового числа $Z_{d}=998$. Для начальной плотности электронов во время ускорения, равной $n_{e_{0}}=10^{10} \mathrm{~cm}^{-3}$, доля ионов и макрочастиц при $t=0$ есть $n_{i 0} / n_{e 0}=1.01$ и $n_{d 0} / n_{e 0}=10^{-5}$ соответственно. Также следует отметить, что в настоящей работе процесс зарядки макрочастиц пылевой плазмы [13,17-19] как таковой не рассматривается, поскольку основной целью работы является качественное рассмотрение принципиальной возможности ускорения потока пылевой плазмы в электромагнитных полях специального вида.

Во всех расчетах постоянное радиальное поле было направлено внутрь плазменного цилиндра, так что $B_{r_{0}}=-1$. Начальные скорости электронов, ионов и макроскопических частиц принимали следующие значения: $A_{e}(0)=A_{i}(0)=-10^{-2}$ и $A_{d}=-7 \cdot 10^{-3}$, т. е. радиальные составляющие скорости были направлены внутрь плазменного цилиндра, $-C_{e}(0)=-C_{d}(0)=C_{i}(0)=10^{-3}$, $D_{e}(0)=D_{i}(0)=D_{d}(0)=10^{-1}$. Начальные значения собственного электрического поля принимались равными $\varepsilon_{r}(0)=0, \varepsilon_{\varphi}(0)=0$ и $\varepsilon_{z}(0)=0$, а начальное значение возмущенного аксиального магнитного поля $B_{z}^{*}(0)=0$.

На рис. 2, $а$ представлена зависимость аксиальных импульсов электронного $D_{e}$, ионного $D_{i}$ потоков и потока макрочастиц $D_{d}$, когда $B_{r 0}=-1$ и $B_{z 0}=-1$, т. е. когда внешнее аксиальное поле постоянно на всем временно́м промежутке ускорения, а на рис. $2, b$ представлены такие же зависимости для $B_{r 0}=-1$ и $B_{z 0}=-0.25 t$, т. е. когда внешнее аксиальное магнитное поле является постоянно убывающей линейной функцией времени.

Также проводилось исследование зависимости аксиальной составляющей скоростей компонент пылевой плазмы от направления радиальной составляющей скорости основного плазменного потока (электронной и ионной компонент). Так, в расчетах принимались следующие значения для радиальных компонент скоростей: $A_{e}(0)=A_{i}(0)=10^{-2}$ и $A_{d}=-7 \cdot 10^{-3}$, т.е. начальная радиальная составляющая скорости пылевой компоненты была все так же направлена в центр цилиндра, а у электронной и ионной компонент плазменного потока направлена наружу. Величины начальных значений азимутальных и аксиальных компонент были следующими: $-C_{e}(0)=-C_{d}(0)=C_{i}(0)=10^{-3}$ и $D_{e}(0)=D_{i}(0)=D_{d}(0)=10^{-1}$. Начальные значения собственного электрического поля принимались равными $\varepsilon_{r}(0)=0, \varepsilon_{\varphi}(0)=0$ и $\varepsilon_{z}(0)=0$, а начальное значение возмущенного аксиального магнитного поля $B_{z}^{*}(0)=0$. В результате были получены зависимости аксиальных импульсов электронов, ионов и макрочастиц (рис. 3). 

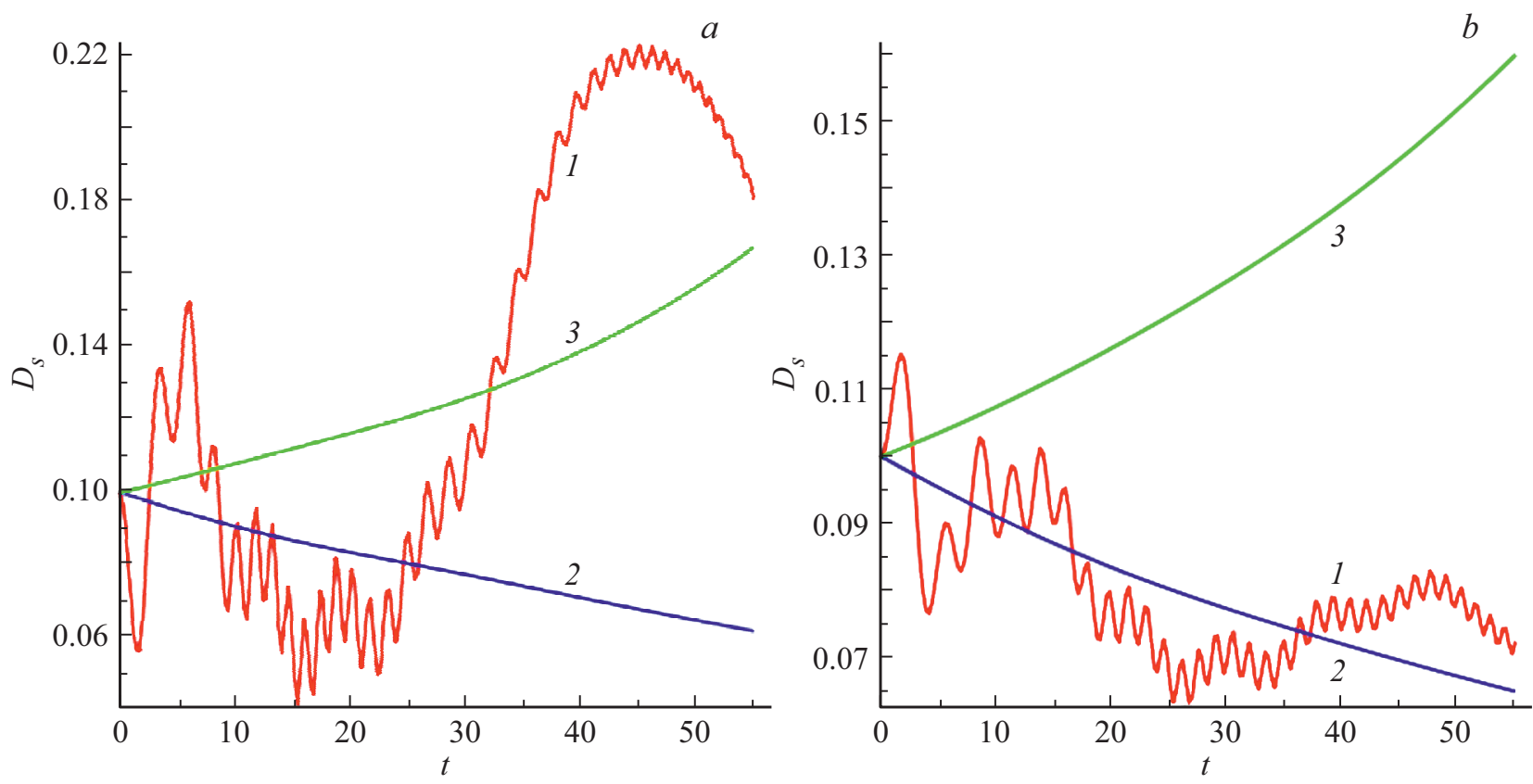

Рис. 3. Временны́е зависимости $D_{e}(1), D_{i}(2)$ и $D_{d}$ (3) для $A_{e}(0)=A_{i}(0)=10^{-2}, A_{d}=-7 \cdot 10^{-3},-C_{e}(0)=-C_{d}(0)=10^{-3}$, $D_{e}(0)=D_{i}(0)=D_{d}(0)=10^{-1}$ в случае $B_{r_{0}}=-1$ и $B_{z_{0}}=-1(a)$ и в случае $B_{r_{0}}=-1$ и $B_{z_{0}}=-0.25 t(b)$.

Существуют различные варианты динамики ускорения макроскопических частиц: в случае, изображенном на рис. 2, все компоненты пылевой плазмы будут ускоряться; в варианте, приведенном на рис. $3, b$, будут ускоряться только массивные многозарядные частицы. При этом величина аксиального импульса пылевых частиц в случае с замедлением электронной и ионной компонент превышает аналогичное значение при одновременном ускорении всех трех компонент, но наиболее сильное влияние на темп ускорения макрочастиц оказывает радиальная компонента скорости.

Таким образом, в рамках упрощенной модели холодного потока пылевой плазмы для схемы ускорения, изображенной на рис. 1 , нам удалось продемонстрировать возможность захвата макроскопических заряженных частиц с последующим их ускорением. Также следует отметить, что результаты по совместному ускорению зарядов разной массы и знаков в рассматриваемой схеме наводят на мысль о возможности ускорения макромолекул с огромными дипольными моментами, что было бы интересно с точки зрения воздействия на структуру сложных полимерных материалов.

\section{Финансирование работы}

Исследование выполнено при финансовой поддержке РФФИ в рамках научного проекта № 19-32-90105.

\section{Конфликт интересов}

Авторы заявляют, что у них нет конфликта интересов.

\section{Список литературы}

[1] Pawlowski L. The science and engineering of thermal spray coatings. N.Y.: Wiley, 1995. $432 \mathrm{p}$.

[2] Жуков Б.Г., Куракин Р.О., Сахаров В.А., Бобамев С.В., Поняев С.А., Резников Б.И., Розов С.И. // Письма в ЖТФ. 2013. T. 39. B. 12. C. 63-70.

[3] Скворцов В.В. Аэродинамические исследования при участии потоков синтезированной и низкотемпературной плазмы. М.: Физматлит, 2013. 218 с.

[4] Бешенков В.Г., Знаменский А.Г., Марченко В.А., Пустовит A.Н., Черных А.В. // ЖТФ. 2007. Т. 77. В. 5. С. 102-107.

[5] Арцимович Л.А., Лукьянов С.Ю., Подгорный И.М., Чуватин С.А. // ЖЭТФ. 1958. Т. 33. В. 1. С. 3-8.

[6] Бобашев С.В., Жуков Б.Г., Куракин Р.О., Поняев С.А., Резников Б.И., Розов С.И. // Письма в ЖТФ. 2010. Т. 36. B. 2. C. 54-61.

[7] Плеханов А.В., Кудрявцев А.В., Железный В.Б., Хандрыга Д.В. // ПМТФ. 1996. Т. 37. № 1. С. 15-20.

[8] Жуков Б.Г., Резников Б.И., Куракин Р.О., Поняев С.А., Бобашев С.В. // ЖТФ. 2016. Т. 86. В. 11. С. 43-49.

[9] Karimov A.R., Murad P.A. // IEEE Trans. Plasma Sci. 2017. V. 45. N 7. Pt 2. P. 1710-1716.

[10] Karimov A.R., Murad P.A. // IEEE Trans. Plasma Sci. 2018. V. 46. N 4. Pt 2. P. $882-887$.

[11] Karimov A.R., Terekhov S.A., Shikanov A.E., Murad P.A. // IEEE Trans. Plasma Sci. 2019. V. 47. N 3. P. 1520-1525.

[12] Цитович В.Н. // УФН. 1997. Т. 167. № 1. С. 57-99.

[13] Фортов В.Е., Храпак А.Г., Храпак С.А., Молотков В.И., Петров О.Ф. // УФН. 2004. Т. 174. № 5. С. 495-544.

[14] Karimov A.R., Yu M.Y., Stenflo L. // J. Plasma Phys. 2013. V. 79. N 6. P. 1007-1009.

[15] Karimov A.R. // Phys. Plasmas. 2013. V. 20. N 5. P. 052305.

[16] Karimov A.R., Yu M.Y., Stenflo L. // J. Plasma Phys. 2016. V. 82. N 5. P. 905820502. 
[17] Shukla P.K., Mamun A.A. Introduction to dusty plasma physics. London: IOP Publ. Ltd, 2002. 284 p.

[18] Tawidian $H$. Formation and behavior of nanoparticles in a plasma: dusty plasma instabilities. Université d'Orléans, 2013. $183 \mathrm{p}$.

[19] Konopka U., Morfill G.E., Ratke L. // Phys. Rev. Lett. 2000. V. 84. N 5. P. $891-894$ 